\title{
Motion artifacts reduction in cardiac pulse signal acquired from video imaging
}

\author{
Murthad Al-Yoonus', Mustafa H. Alhabib², Mustafa Zuhaer Nayef Al-Dabagh ${ }^{3}$, M. F. L. Abdullah ${ }^{4}$ \\ ${ }^{1}$ Computer and Networks Department, Paitaxt Technical Private Institute- Erbil, KRG, Iraq \\ ${ }^{2}$ Communication and Computer Engineering Department, Cihan University-Erbil, KRG, Iraq \\ ${ }^{3}$ Department of Computer Science, College of Science, Knowledge University, \\ University Park, Erbil EBL 446015, Kurdistan Region, Iraq \\ ${ }^{1,4}$ Faculty of Electrical and Electronic Engineering, Universiti Tun Hussein Onn Malaysia, Malaysia
}

\section{Article Info}

Article history:

Received Aug 4, 2019

Revised Apr 13, 2020

Accepted May 8, 2020

\section{Keywords:}

Cardiac pulse rate

FFT

Illumination

Low-pass filter

Motion artifacts

Pulse oximeter

\begin{abstract}
This study examines the possibility of remotely measuring the cardiac pulse activity of a patient, which could be an alternative technique to the classical method. This type of measurement is non-invasive. However, several limitations may deteriorate the accuracy of the results, including changes in ambient illumination, motion artifacts (MA) and other interferences that may occur through video recording. The paper in hand presents a new approach as a remedy for the aforementioned problem in cardiac pulse signals extracted from facial video recordings. Partitioning provides the basis for the presented MA reduction method; the acquired signals are partitioned into two sets for each second and every partition is shifted to the mean level and then all the partitions are recombined again into one signal, which is followed by low-pass filtering for enhancement. The proposed compared with ordinary pulse oximetry Photoplethysmographic (PPG) method. The resulted correlation coefficient was found (0.957) when calculated between the results of the proposed method and the ordinary one. Experiments were implemented using a common camera by creating a dataset from 11 subjects. The ease of implementation of this method with a simple that can be used to monitor the cardiac pulse rates in both home and the clinical environments.
\end{abstract}

Copyright @ 2020 Institute of Advanced Engineering and Science. All rights reserved.

Corresponding Author:

Murthad Al-Yoonus,

Computer and Networks Department,

Paitaxt Technical Private Institute-Erbil,

100 Street, Erbil, KRG, Iraq.

Email: eng.murthad86@gmail.com

\section{INTRODUCTION}

The Measuring and monitoring of human cardiac pulse activity are an essential topic for the healthcare industry in both clinical and home environments. Currently, Photoplethysmographic (PPG) based pulse oximetry and electrocardiography (ECG) represent the most common techniques being used in modern cardiac pulse signal monitoring systems, which are both inexpensive and reliable. However, these methods use contact sensors that have to be attached to the body, which poses a disadvantage since these sensors are wired to the measuring equipment, making the process of placement and removal inconvenient to the patient. To overcome the pre-stated problem of skin-attached sensors, some recent studies illustrated a number of innovative contactless solutions that measure cardiac pulse rates using a camera [1-7].

The camera is used to record facial videos while the cardiac pulse activity is obtained by further signal processing analysis for acquiring the heart beat activity [8]. This kind of contactless technique for cardiac pulse parameters monitoring can become an essential tool for hospital health care applications as well as in the home environment. These novel techniques use a similar principle to pulse oximetry as a contact 
PPG, where the changes in skin color reflects the variations in blood volume according to the cardiovascular events $[9,10]$. In other words, the heart pulse activities affect light transmission and reflectance from the human skin. Pulse oximetry PPG is based in the transmission mode, while heart rate measuring by using a camera is based on the reflectance mode [11-14].

However, the later method still faces some challenges; including motion artifacts, illumination changes, and other interferences that may occur during video recording. Over the years, various kinds of literature proposed signal processing algorithms to accommodate for artifact interference and noise cancellation to enhance the signals obtained from the PPG sources. Some of these methods have been proposed for reducing the interference caused by motion artifacts [15-17]. Many algorithms relied on independent component analysis (ICA) for decomposing the PPG signals in order to obtain the desired heart rate signal $[18,19]$. Another study exploited the hilbert-huang transform (HHT) as a decomposition method for reducing the motion of artifact effects in Photoplethysmographic signals. Here, the intrinsic mode function (IMF) components are obtained from nonlinear data by applying empirical mode decomposition, which is also used for exploring the changes in amplitude over time [20]. Also, a comparative analysis is presented between the performance of adaptive filtering techniques and wavelet transform. The aim was at acquiring PPG signals that enjoy minimum artifacts effect to be used to approximate the cardiac pulse rate. In this case, only limited application can be driven from motion reduction-based wavelet transform method [21].

Another comparison can be seen in [22] where different motion artifacts were processed by different wavelet transform techniques for the possibility of reduction. The implementation of different wavelets on PPG signals yielded $\mathrm{SpO}_{2}$ estimates with only slight variations, while adaptive filtering has been used to reduce the effects of motion artifacts on the PPG signal. A verity of different approaches where implemented together to achieve the same task, such as adaptive comb filter (ACF), infinite impulse response (IIR) and adaptive lattice notch filter (ALNF) such as in [23]. Alternatively, the work in [24] suggested a two-stage method for motion artifacts reduction: in the first stage, the inherent time and frequency domain (FD) characteristics of the PPG signal are used to detect motion artifacts while the second stage applied signal processing enhancement featuring a new magnitude algorithm based on an FD-ICA approach [24].

The study presented in [25] proposed a multi-wavelength motion artifacts reduction algorithm framework for wrist-worn PPG sensors, utilizing the green component of the PPG signal for heart rate monitoring in addition to allocating an infrared PPG signal as the motion reference. The authors presented four main steps for system realization: Motion detection, motion removal using continuous wavelet transform, approximate HR estimation and signal reconstruction.

The algorithm presented in this paper demonstrates a new method for motion artifacts reduction using a remotely-obtained Photoplethysmograph (rPPG) signal from facial video recordings of eleven participants using face detection algorithm [26]. This method can measure the cardiac pulse rate of a person from a short distance up to two meters by using a camera connected to a PC. This is followed by using the MATLAB (MathWorks ${ }^{\mathrm{TM}}$ ) software for processing the signal data (signal extraction and filter enhancement for motion artifacts reduction). Finally, the enhanced signal with the frequency of the cardiac pulse is displayed as results.

Eleven subjects had participated in the experiments for recording 30-second videos for each individual. The contribution of this research can be summarized as follows:

- The extraction of the cardiac pulse signal from the green channel in facial videos, (using the color RGB separation methods stated in the methodology section).

- Partitioning the green component into 60 partitions (two partitions per a second).

- Partitions Enhancement.

- Recombining the enhanced partitions into one signal.

- To find the cardiac pulse rate frequency, fast fourier transform (FFT) is applied to get the desired signal.

The arrangement of this research will be as follows: Section 2 describes the proposed method for cardiac pulse signal detection and motion artifacts reduction using the green component of the PPG signal. Section 3 presents the experimental results and explains the effectiveness of the proposed method, regarding noise reduction in the cardiac pulse signals obtained from video recordings. Finally, Section 4 discusses the results, presents the conclusion and offers future recommendations.

\section{METHOD}

\subsection{Experimental setup}

In this research, eleven individuals ranging between 18-35 years old were participated for acquiring the PPG signals. An ordinary camera is used to record the facial videos in true color standard (24-bit per pixel RGB format with 3 channels $\times 8$ bits/channel) at 30 frames per second (fps), with a pixel resolution of $720 \times 404$. The videos were saved in AVI format for further analysis using MATLAB software. The recording 
sessions took place at different times of the day. A varying amount of ambient sunlight was the only source of illumination in this experiment. The participants, having different skin colors, were seated on a chair in front of the camera at a distance of approximately one meter. During the recording, participants were asked to sit still and stare at the camera lens.

\subsection{Cardiac pulse signal extraction}

The signal of interest for this work is the cardiovascular pulse wave that propagates throughout the body. The Viola-Jones algorithm provides the basis for the region of interest (ROI) selection for face detection. The camera senses the changes in the amount of reflected light, which indicates the timing of cardio pulse events. These events relate to volumetric changes in the ROI blood vessels during the cardiac cycle. The RGB channels for each frame consist of three components; these are $r(t)$, $g(t)$ and $b(t)$ for red, green and blue respectively. The extracted signal from the green color sensor is the best signal to show the cardiac activity, being attenuated by motion artifact noise.

The presumption is that the volumetric blood variations result in facial color changes and hence facial light intensity variations. Figure 1 shows the PPG signals being acquired from the facial video recording measurements of two participants by using a camera connected to a PC at different times of the day. The observed small ripple in the green component is according to the cardiovascular events; however, the red line indicates the significant amplitude changes resulted from undesirable motion artifacts. The signals obtained from these green components show evident cardiac pulse characteristics and accommodates the motion of artifacts, such as small facial motion and illumination changes. The aim here is at reducing motion artifacts to enhance this signal and only yield the clear cardiac pulse signal.

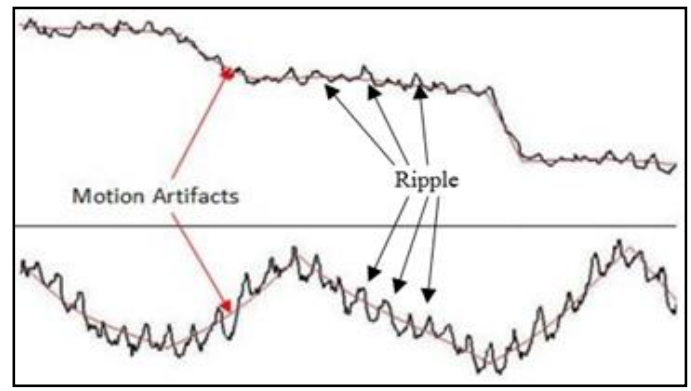

Figure 1. Two PPG cardiac pulse signals with motion artifacts noiseof two participants, the signals were taken at a distance 1 meter

\subsection{Motion artifacts reduction}

Signal enhancement and motion artifacts elimination requires the implementation of a new method which is based on partitioning the signal, shifting partitions to the mean level, recombining them and then applying low-pass filter (LPF) to obtain the final signal. Figure 2 shows the acquired signal as well as the enhancement processes.

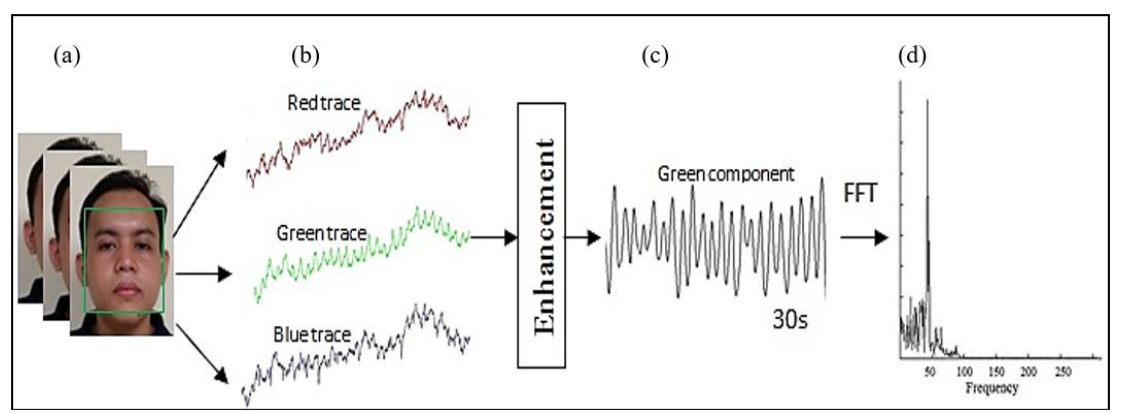

Figure 2. (a) The (ROI) is automatically cropped using face detection, (b) the ROI decomposes into the RGB channels and the raw RGB traces, (c) the green component is enhanced,

(d) FFT is applied to recover the frequency 
The standard cardiac pulse rate of an adult ranges from 60 to 100 beats per minute; meaning that every second contains one or two pulses according to the participant's health condition. The partitioned signal is obtained from the green component over 30 seconds and is divided into 60 partitions (two partitions per second) to separate every possible pulse in one partition and then shift partitions to the mean level. Then all partitions are recombined again to form the signal. Lastly, using a $4 \mathrm{~Hz}$ low-pass filter (LPF) will result in the final enhanced signal. The following steps are applied for reducing the effect of motion artifacts in cardiac pulse signals obtained from a video recording of the human face:

- Partitioning the green component into 60 partitions as in Figure 3(a).

- Get the mean level for every partition as shown in the formula below:

$$
\bar{X}=\frac{\sum_{i=1}^{n} g(t)_{i}}{n}
$$

where $n$ represents the No. partitions

- Shift the samples of every partition to the mean level as in Figure 3(b)., using:

$$
G(t)=g(t)-\bar{X}
$$

- Repeat step 3 for all partitions, then recombine all the partitions to yield the clear cardio pulse signal with the reduction of artifacts as in Figure 3(c).

- Use low-pass filter iteration (4 times) of order $4 \mathrm{~Hz}$ for the final step of enhancement, Figure 3(d).

The processing of data requires the transformation into the frequency domain (FD) by Fast Fourier. Transform, which will estimate the frequency of the cardiac pulse to facilitate signal enhancement. At the same time, a pulse oximeter measures the reference of the cardiac pulse rate. This pulse oximeter data acts as ground truth that enables accuracy comparison and assessment of the camera algorithm-based measurement method.

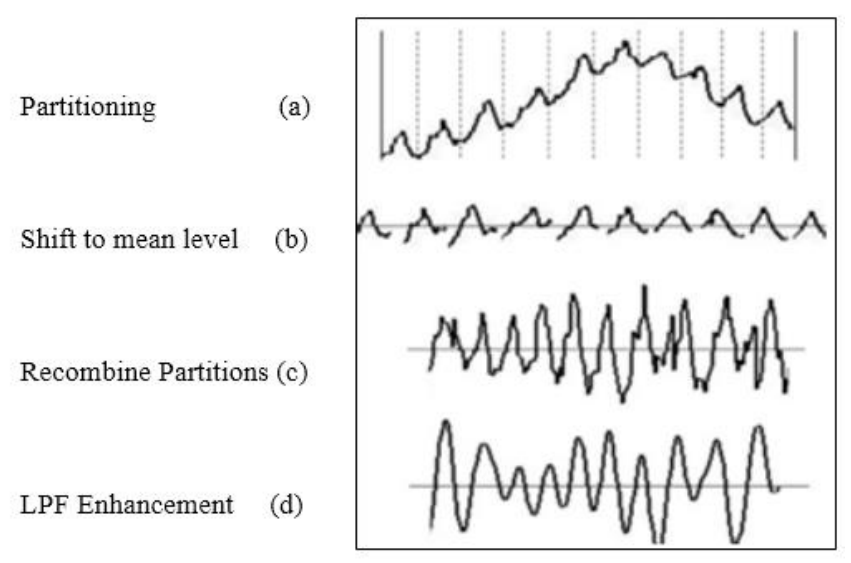

Figure 3. (a) Partitioning, (b) shifting partitions to the mean, (c) recombining the Partitions, (d) LPF Enhancement

\section{EXPERIMENTAL RESULTS}

In the experiments, the camera was adjusted to be about 1meter away from the subject to collect RGB data weight variations during video recording. The experiments were performed indoor and outdoor at different times of the day. The faces of the subjects are considered to be the region of interest, being cropped for analysis after face detection. From the experiments, the green sensor collected the best data where weight variations correspond to cardiovascular activities. Also, the presence of other fluctuation sources in light with the obtained data will introduce noise to the signal. The motion artifacts reduction method was applied on the obtained signal using MATLAB software.

Figure 4 shows the typical results of the cardiac pulse remote measurement and motion artifacts reduction obtained by this method for three subjects Figure 4(a) shows the signals in time domain with different amplitudes caused by light intensity variations according to the cardiovascular events and motion artifacts. Figure 4(b) shows the results of the artifacts reduction method by partitioning, shifting to a mean 
level, recombining and then applying a low-pass filter iteratively for each case. Figure 4(c) shows the FFT of the enhanced signals in frequency domain, the FFT spectra clearly show a higher peak; this peak is at the frequency of the cardiac pulse.

In order to test the heart rate measurement accuracy of this method for motion artifact reduction, 11 subjects participated in the experiments. The results of this new heart rate measuring method compare favorably with the reference results previously obtained from a pulse oximeter as shown in Table 1 . This method produces a stable correlation coefficient of (0.957) calculated between the cardiac pulse rate measurements acquired here and the classical pulse oximeter method, as shown in Figure 5.

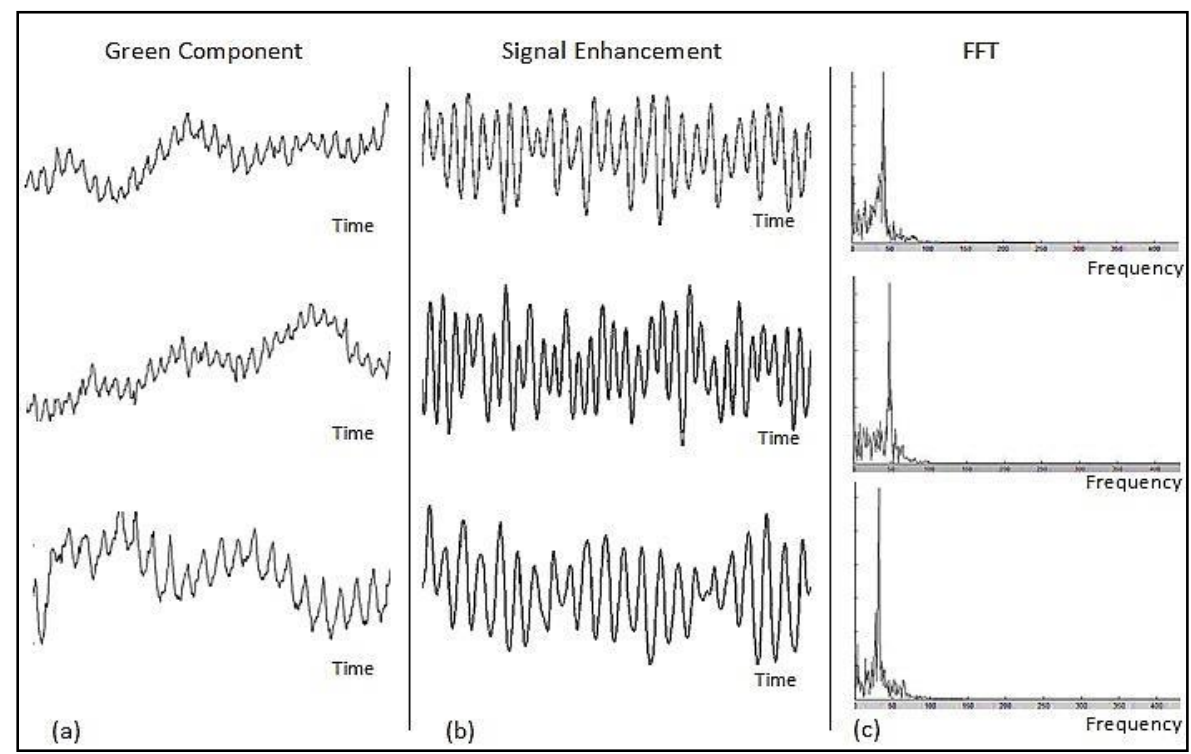

Figure 4. (a) Signals in time domain with different amplitudes, (b) artifacts reduction, (c) high pecks at cardiac pulse rate in frequency domain

Table 1. Cardiac pulse rate results compared with reference results

\begin{tabular}{ccc}
\hline Subjects & Proposed Method & Pulse Oximeter \\
\hline 1 & 55 & 58 \\
2 & 72 & 76 \\
3 & 63 & 63 \\
4 & 88 & 86 \\
5 & 83 & 81 \\
6 & 60 & 57 \\
7 & 60 & 64 \\
8 & 95 & 83 \\
9 & 86 & 79 \\
10 & 56 & 58 \\
11 & 69 & 69 \\
\hline
\end{tabular}

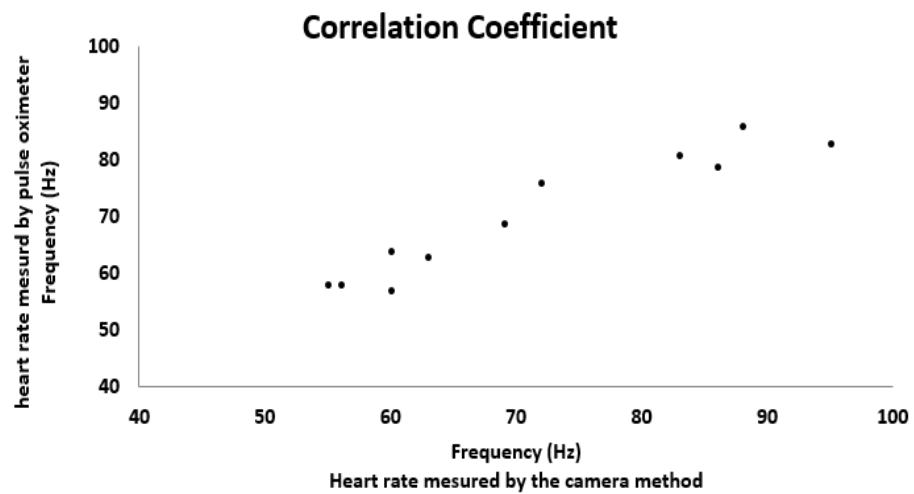

Figure 5. The cardiac pulse rate correlation coefficient between the camera method and the pulse oximeter 


\section{DISCUSSION AND CONCLUSION}

This paper presents a technique for motion artifacts reduction in cardiac pulse signals obtained by remote video recording. This method consists of two stages: firstly, partitioning the signal, shifting the partitions to the mean level and recombining them back into one signal; the second stage is signal enhancement by low-pass filtering. In order to test the performance of this artifacts reduction method, the applied FFT enhances the cardiac pulse rate by identifying high peaks in the frequency domain.

The remote pulse measurement results compare favorably to the results obtained from a regular pulse oximeter. The correlation coefficient between the results of the two methods is found to be $(0.957)$. The technique can reduce the effect of motion artifacts in cardiac pulse PPG for signal enhancement. Furthermore, this study demonstrates that this method can work efficiently with videos recorded at different times of the day. This technique is currently under development but it can be utilized later for building a robust, remote, accurate cardiac pulse rate measuring systems. Topics for future research may include: creating a real-time remote measuring system based on this method for multi-parameters such as cardio pulse rate variability, respiratory and arterial blood oxygen saturation.

\section{REFERENCES}

[1] Poh, Ming-Zher, Daniel J. McDuff, and Rosalind W. Picard, "Non-contact, automated cardiac pulse measurements using video imaging and blind source separation," Optics express, vol. 18, no. 10, pp. 10762-10774, 2010.

[2] Garbey, et al., "Contact-free measurement of cardiac pulse based on the analysis of thermal imagery," IEEE Transactions Biomedical Engineering, vol. 54, no. 8, pp. 1418-1426, 2007.

[3] Cennini, et al., "Heart rate monitoring via remote photoplethysmography with motion artifacts reduction," Optics express, vol. 18, no. 5, pp. 4867-4875, 2010.

[4] Lewandowska, M., Rumiński, J., Kocejko, T., and Nowak, J., "Measuring pulse rate with a webcam-a non-contact method for evaluating cardiac activity," Federated Conference Computer Science and Information Systems (FedCSIS), pp. 405-410, 2011.

[5] Takano, and Yuji Ohta, "Heart rate measurement based on a time-lapse image," Medical engineering and physics, vol. 29, no. 8, pp. 853-857, 2007.

[6] Li, X., Chen, J., Zhao, G., and Pietikainen, M, "Remote heart rate measurement from face videos under realistic situations," Proceedings of the IEEE conference on computer vision and pattern recognition, pp. 4264-4271, 2014.

[7] Rouast, P. V., Adam, M. T., Chiong, R., Cornforth, D., Lux, E., "Remote heart rate measurement using low-cost RGB face video: a technical literature review," Frontiers of ComputerScience, vol. 12, no. 5, pp. 858-872, 2018.

[8] Ibrahim, W., Tomari, R., Zakaria, W. N. W., and Othman, N, "Non-contact heart rate monitoring analysis from various distances with different face regions," International Journal of Electrical and Computer Engineering (IJECE), vol. 7, no. 6, pp. 3030-3036, 2017.

[9] Hassan, et al., "Heart rate estimation using facial video: A review," Biomedical Signal Processing and Control, vol. 38, pp. 346-360, 2017.

[10] N. Ibrahim, et al., "Analysis of minimum face video duration and the effect of video compression to image-based non-contact heart rate monitoring system," Bulletin of Electrical Engineering and Informatics, vol. 9, no. 1, pp. 403-410, 2020.

[11] Aarts, et al.,"Non-contact heart rate monitoring utilizing camera photoplethysmography in the neonatal intensive care unit-A pilot study," Early human development, vol. 89, no. 12, pp. 943-948, 2013.

[12] Al-Naji, and Javaan Chahl, "Noncontact heart activity measurement system based on video imaging analysis," International Journal of Pattern Recognition and Artificial Intelligence, vol. 31, no. 2, pp. 1757001, 2017.

[13] Alghoul, et al., "Heart rate variability extraction from videos signals: ICA vs. EVM comparison," IEEE Access, vol. 5, pp. 4711-4719, 2017.

[14] MasnaniBt Mohamed, et al., "Non Contact monitoring of heart rate responses to taste stimuli using a video camera," Indonesian Journal of Electrical Engineering and Computer Science, vol. 18, no. 1, pp. 293-300, 2020.

[15] Tanweer, et al., "Motion artifact reduction from PPG signals during intense exercise using filtered X-LMS," IEEE International Symposium on Circuits and Systems (ISCAS), pp. 1-4, 2017.

[16] Dao, et al., "A robust motion artifact detection algorithm for accurate detection of heart rates from photoplethysmographic signals using time-frequency spectral features," IEEE journal of biomedical and health informatics, vol. 21, no. 5, pp. 1242-1253, 2016.

[17] Nordin, W. David Hairston, and Daniel P. Ferris, "Dual-electrode motion artifact cancellation for mobile electroencephalography," Journal of neural engineering, vol. 15, no. 5, 2018.

[18] Y. Jianchu, and S. Warren, "A Short Study to Assess the Potential of Independent Component Analysis for Motion Artifact Separation in Wearable Pulse Oximeter Signals," IEEE Conference of the Engineering in Medicine and Biology Society, pp. 3585-3588, 2005.

[19] B. S. Kim, and Sun K. Yoo, "Motion artifact reduction in photoplethysmography using independent component analysis," IEEE Trans. Biomed. Eng., vol. 53, no. 3, pp. 566-568, 2006.

[20] Raghuram, et al., "HHT based signal decomposition for reduction of motion artifacts inphotoplethysmographic signals," Instrumentation and Measurement Technology Conference (I2MTC), pp. 1730-1734, 2012. 
[21] Foo and Jong Yong A, "Comparison of wavelet transformation and adaptive filtering in restoring artifact-induced time-related measurement," Biomed. Signal Process. Control, vol. 1, pp. 93-98, 2006.

[22] Raghuram, et al., "Evaluation of wavelets for reduction of motion artifacts inphotoplethysmographic signals," 10th International Conference Information Sciences Signal Processing and their Applications (ISSPA), pp. 460-463, 2010.

[23] Lee, et al., "Adaptive comb filtering for motion artifact reduction from PPG with a structure of adaptive lattice IIR notch filter," Annual International Conference Engineering in Medicine and Biology Society, pp. 7937-7940, 2011.

[24] Krishnan, et al., "Two-stage approach for detection and reduction of motion artifacts in photoplethysmographic data," IEEE Transactions Biomedical Engineering, vol. 57, no. 8, pp. 1867-1876, 2010.

[25] Zhang, et al., "Motion Artifact Reduction for Wrist-Worn Photoplethysmograph Sensors Based on Different Wavelengths," Sensors, vol. 19, no. 3, 1-18, 2019.

[26] Viola, and Michael Jones, "Rapid Object Detection using a Boosted Cascade of Simple Features," IEEE Computer Society Conference on Computer Vision and Pattern Recognition, vol. 1, pp. 511-518, 2001.

\section{BIOGRAPHIES OF AUTHORS}
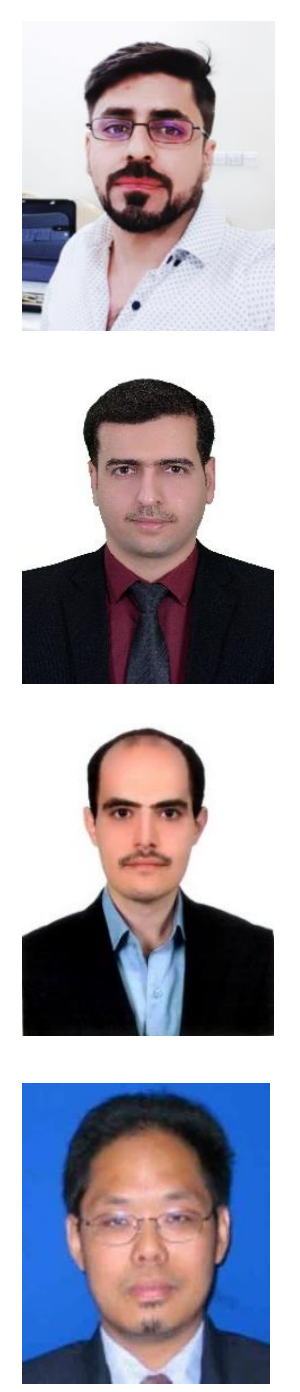

Murthad Al-Yoonus received a M.Eng in Electronic and Computer Engineering from University Technical Melaka Malaysia, he worked in Al Kunooze University College 20162019, teaching and supervision of graduated Projects. Currently lecturing at Paitaxt Technical Private Institute, Erbil, KRG of Iraq. His research interests include Image, signal processing, deep machine learning and Mobile Robotic System. Published four papers in the fields of Image Processing and pattern recognition.

Mustafa H. Mohammed Alhabib completed BSc. degree from the Department of Computer Engineering, College of Engineering, University of Mosul in 2008, obtained MSc degree in Technical Computer Engineering from the Technical Engineering College Mosul in 2013. Specialized in Image Processing and Artificial Intelligence fields. Currently lecturing at the department of Communications and Computer Engineering at cihan University, Erbil, KRG of Iraq. Published 5 papers in the fields of Medical Image Processing and pattern recognition.

Mustafa Z. Nayef Al-Dabagh received the B.Sc. and M.Sc degree in Computer Engineering Technology from Northern Technical University, Iraq in 2008 and 2014. He is working as a lecturer on Knowledge university. His current research interests include Image Processing, Pattern Recognition, and Machine Learning, Biomedical Engineering, and Robotics Systems.

M. F. L. Abdullah He received Phd degree Electrical Engineering from (University Warwick), Master Electrical Engineering (University Technology Malaysia), BSc Electrical Engineering (University Technology Malaysia), Dip Edu (UTM), Certificate Electronic Engineering (Polytechnic Ungku Omar). Currently lecturing at the Department of Communication Engineering, Universiti Tun Hussein Onn Malaysia. Published 153 papers in the field of Electrical and Communication, signal processing and fiber optics. 